\title{
DIAGNOSTIC ACCURACY OF YALE OBSERVATIONAL SCALE IN BACTEREMIA IN INFANTS AND CHILDREN.
}

1. MBBS, FCPS Senior Registrar Children Hospital Faisalabad.

2. MBBS, FCPS

Associate Professor Paediatrics Faisalabad Medical University.

3. MBBS, FCPS

Assistant Professor Paediatrics Children Hospital Faisalabad.

4. MBBS, FCPS

Assistant Professor Pediatrics Faisalabad Medical University.

5. MBBS, FCPS

Professor of Paediatrics

Faisalabad Medical University.

Correspondence Address:

Dr. Zahid Mahmood Anjum

Department of Paediatrics

252-J Tech Town, Satiana Road,

Faisalabad.

zmahmoodch@Yahoo.com

Article received on:

$14 / 03 / 2019$

Accepted for publication:

$12 / 06 / 2019$

\section{INTRODUCTION}

Bacteremia is the presence of bacteria in the blood stream. It may result from ordinary activities such as vigorous tooth brushing, dental or medical procedures. Typically bacteremia causes no symptoms because bacteria are in small number and rapidly removed from the blood steam by the immune system. However if bacteria are present long enough and in large enough numbers, particularly in people who have a weekend immune response, can accumulate in certain tissues or organs and cause serious bacterial infections like pneumonia, meningitis, brain abscess and sepsis. ${ }^{1-3}$

Blood culture in which a sample of blood is allowed to incubate with a medium that promotes bacterial growth is standard diagnostic tool. ${ }^{4}$ In Pakistan where $70 \%$ of population is based in rural areas and lack modern health facilities. It is difficult to obtain a blood culture in such health facilities. Detailed clinical examination needs time \& expertise and may not yield clinical signs leading to clue to diagnosis especially in young children. ${ }^{5}$ Yale Observation Scale (YOS) is a scale consisting of six observational items originally characterized and clubbed by McCarthy et al11 in 1982, and is validated in young febrile children to detect serious illness. It is simple, quick, easy to apply and cost-effective, as it is purely observational and does not contain investigational items. ${ }^{6}$ In a study, the sensitivity and specificity of YOS score of more than 10 to predict bacteremia was $87.93 \%$ and $83.78 \% .{ }^{7}$ Bacteremia was found in $28.06 \%$ patients. $^{7}$

The study was carried out to determine diagnostic accuracy of the Yale Observation Scale (YOS) for diagnosing bacteremia, taking blood culture as gold standard. Moreover, it will also help us to design a protocol for early screening and diagnosis of bacteremia in infants which will help the clinicians for starting early treatment of these particular patients, so that patient's morbidity and 
mortality can be minimized.

\section{MATERIAL \& METHODS Objectives of Study}

The objective of my study was to determine the diagnostic accuracy of Yale observation scale (YOS) for diagnosing bacteremia taking blood culture as gold standard. It was a Cross sectional (validation) study conducted Department of Pediatric Medicine unit II, District Headquarters Hospital, Faisalabad during $26^{\text {th }}$ May, 2016 to $25^{\text {th }}$ November, 2016.

The Sample Size was calculated By using sensitivity and specificity sample size calculator. Sensitivity $=87.93 \% 7$, Specificity $=83.78 \% 7$, Prevalence $=28.06 \% 7$, Precision for sensitivity $=10 \%$, Precision for specificity $=10 \%$, Confidence level=95\%, Sample size was 105 .

The Sampling Technique used was Non-probability, consecutive sampling.

\section{Inclusion Criteria}

All patients with suspicion of bacteremia (as peroperational definition).

Age 3-36 months.

Both genders.

\section{Exclusion Criteria}

Patients taking antibiotics for fever before admission or who developed fever after admission in hospital.

Febrile illness suggestive of viral infection or malaria or if their siblings illness is of viral origin.

Siblings suffering from viral infections in last 10 days.

Familiar congenital disorders like ectodermal dysplasia and familial dysautonomia.

After permission from local ethical review committee, a total of 105 patients were selected for study fulfilling the inclusion/exclusion criteria. After taking informed consent from patient's parents, Yale Observation score was calculated (as per-operational definition) and bacteremia (present/absent) was noted. After this blood sample of each patient was sent to the institutional pathology laboratory for culture and report was noted as presence or absence of bacteremia. YOS findings was compared with the blood culture report. Data was recorded on proforma by myself.

Bacteremia: All patients presenting with fever (rectal temperature $>38 \mathrm{C}$ o for $8 \mathrm{hrs}$. at least) and Yale Observation Scale $>10$ was taken as positive.

Yale Observation Scale (YOS): it was measured as follows;

Yale Observation Scale (YOS) > 10 was taken as positive for bacteremia.

Bacteremia on blood culture: presence of bacteria in the bloodstream of the patient will be taken as positive when a single bacterial colony be found.

True Positive

Patients with bacteremia on Yale Observation Scale as well as on blood culture.

True Negative

Patients with no bacteremia on Yale Observation Scale as well as on blood culture.

False Positive

Patients with bacteremia on Yale Observation Scale but not on blood culture.

False Negative

Patients with no bacteremia on Yale Observation Scale but present on blood culture.

Diagnostic Accuracy Will be measured in terms of;

Sensitivity which Measures the proportion of positives that are correctly identified as such. Specificity which Measures the proportion of negatives that are correctly identified as such. Positive Predictive Value (PPV) Is the probability that subjects with positive screening test truly have the disease. 


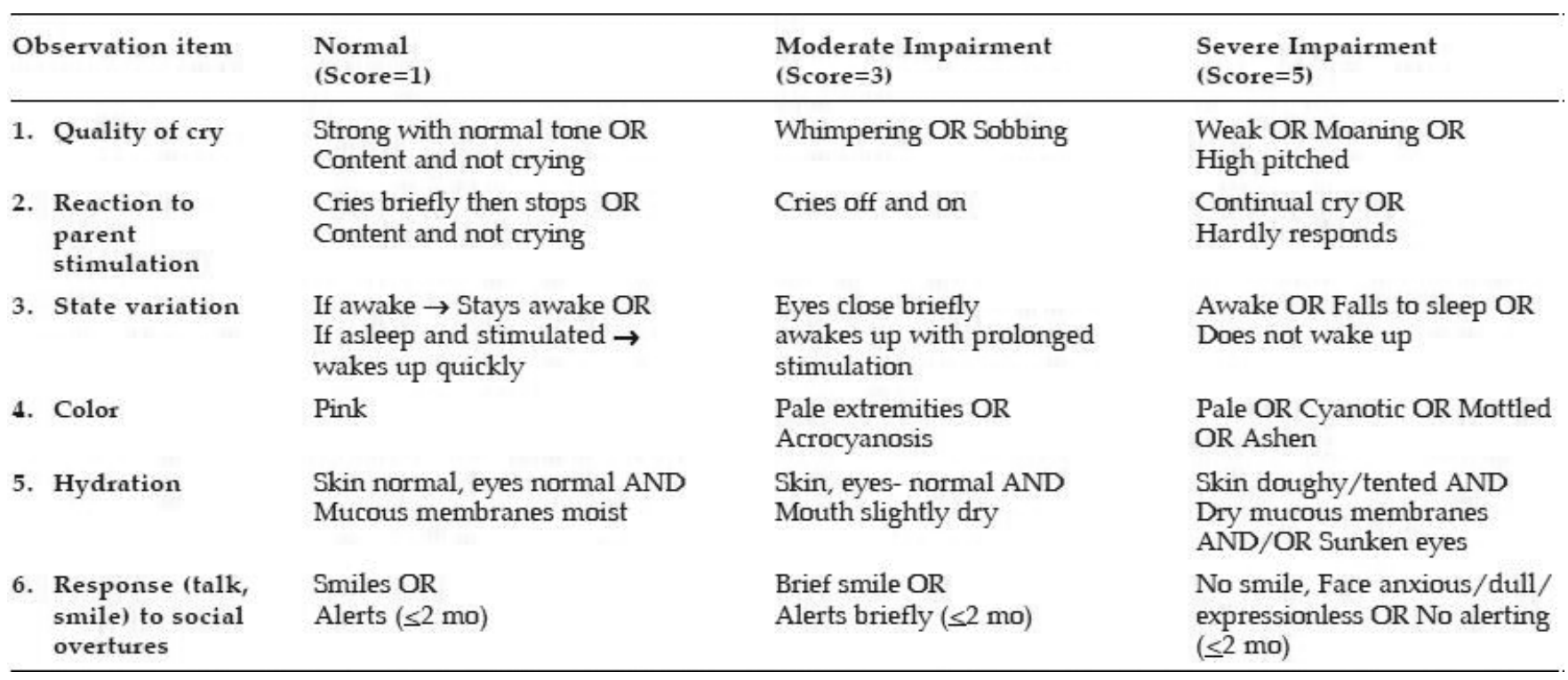

Negative Predictive Value is the probability that subjects with negative screening test truly don't have the disease.

Collected data was analyzed through computer software SPSS 20.0. Mean and standard deviation was calculated for quantitative variables i.e. age and duration of fever. Frequency and percentage was calculated for qualitative variables i.e. gender and bacteremia (present/absent) on YOS and blood culture. $2 \times 2$ contingency table was used to calculate sensitivity, specificity, positive predictive value, negative predictive value and diagnostic accuracy of Yale observation scale in diagnosing bacteremia, taking blood culture as gold standard.

\begin{tabular}{|c|c|c|}
\hline & $+\mathrm{VE}$ & $-V E$ \\
\hline$+\mathrm{VE}$ & $\mathrm{a}(\mathrm{TP})$ & $\mathrm{b}(\mathrm{FP})$ \\
\hline - VE & $\mathrm{c}(\mathrm{FN})$ & $\mathrm{d}(\mathrm{TN})$ \\
\hline
\end{tabular}

Blood Culture

Sensitivity $=\frac{a}{a+c} \times 100$.

Specificity $=\frac{d}{b+d} \times 100$.

Positive Predictive Value (PPV) $=\frac{a}{a+b} \times 100$.
Negative Predictive Value $==^{\frac{\mathrm{d}}{\mathrm{c}+\mathrm{d}}} \times 100$.

Diagnostic Accuracy $=\frac{a+d}{a+b+c+d} \times 100$.

Effect modifiers like age, gender and duration of fever were controlled by stratification. Poststratification diagnostic accuracy was applied to see their effect on outcome and p-value $\leq 0.05$ was considered as significant. ROC and likelihood ratio were calculated.

\section{RESULTS}

One hundred and five patients of age group 3-36 months having fever $>380 \mathrm{C}$ were included in the study.

Out of 105 patients, mean age of the patients was $12.9 \pm 8.24$ months. Minimum age was 3 months while maximum age was 36 months. Out of 105 patients, mean duration of fever was $5.61 \pm 2.087$ days. Minimum duration of fever was 1 day while maximum duration of fever was 9 days.

Out of 105 patients, there were 56 (53.3\%) patients had age $<1$ year, $37(35.2 \%)$ patients had age between 1-2 years and12 (11.4\%) patients had age $>2$ years.

Out of 105 patients, there were 55 (52.4\%) were male and 50 (47.6\%) patients were females. 
Out of 105 patients, there were 53 (50.5\%) patients had $\leq 5$ days of duration of fever and 52 (49.5\%) patients had $>5$ days of duration of fever.

Sensitivity of YOS in diagnosing bacteremia, positive predictive value, negative predictive value and diagnostic accuracy is as shown in (Table-I).

Sensitivity of YOS in diagnosing bacteremia in different age groups as shown in (Table-II)

Sensitivity of YOS in diagnosing bacteremia in male patients came out $100 \%$, specificity $79.31 \%$, positive predictive value $57.14 \%$ and negative predictive value $100 \%$ while diagnostic accuracy came out $83.78 \%$. Sensitivity of YOS in diagnosing bacteremia in female patients came out $100 \%$, specificity $77.78 \%$, positive predictive value $60 \%$ and negative predictive value $100 \%$ while diagnostic accuracy came out $83.33 \%$.

Sensitivity of YOS in diagnosing bacteremia in patients according to duration of fever is shown in (Table-III).

ROC curve is presented in Figure-1.

Area under curve was 0.562 with 0.041 standard error. Table-IV.

\begin{tabular}{|c|c|c|c|c|}
\hline \multicolumn{5}{|c|}{ Blood Culture } \\
\hline \multirow{3}{*}{$\begin{array}{l}\text { Yale Observation Scale } \\
(\text { YOS } \mid)\end{array}$} & Positive & 29 & 15 & 44 \\
\hline & Negative & 3 & 58 & 61 \\
\hline & Total & 32 & 73 & 105 \\
\hline
\end{tabular}

\begin{tabular}{|c|c|c|c|c|c|}
\hline \multirow{2}{*}{ Age Distribution } & \multirow{2}{*}{$\begin{array}{c}\text { Bacteremia on } \\
\text { YOS }\end{array}$} & \multicolumn{2}{|c|}{ Bacteremia on blood culture } & \multirow{2}{*}{ Total } & \\
\hline & & Yes & No & & \\
\hline \multirow{3}{*}{$<1$ year } & yes & 18 & 7 & 25 & \multirow{3}{*}{$\begin{array}{c}\text { Sensitivity }=85.71 \% \\
\text { Specificity }=80 \% \\
\mathrm{PPV}=72 \% \\
\mathrm{NPV}=90.32 \% \\
\text { Diagnostic accuracy }=82.14 \%\end{array}$} \\
\hline & no & 3 & 28 & 31 & \\
\hline & Total & 21 & 35 & 56 & \\
\hline \multirow{3}{*}{ 1-2 year } & yes & 8 & 6 & 14 & \multirow{3}{*}{$\begin{array}{c}\text { Sensitivity }=100 \% \\
\text { Specificity }=79.31 \% \\
\text { PPV }=57.14 \% \\
\text { NPV }=100 \% \\
\text { Diagnostic accuracy }=83.78 \%\end{array}$} \\
\hline & no & 0 & 23 & 23 & \\
\hline & Total & 8 & 29 & 37 & \\
\hline \multirow[t]{3}{*}{$>2$ year } & yes & 3 & 2 & 5 & \multirow{3}{*}{$\begin{array}{c}\text { Sensitivity }=100 \% \\
\text { Specificity }=77.78 \% \\
\text { PPV }=60 \% \\
\text { NPV }=100 \% \\
\text { Diagnostic accuracy }=83.33 \%\end{array}$} \\
\hline & no & 0 & 7 & 7 & \\
\hline & Total & 3 & 9 & 12 & \\
\hline
\end{tabular}

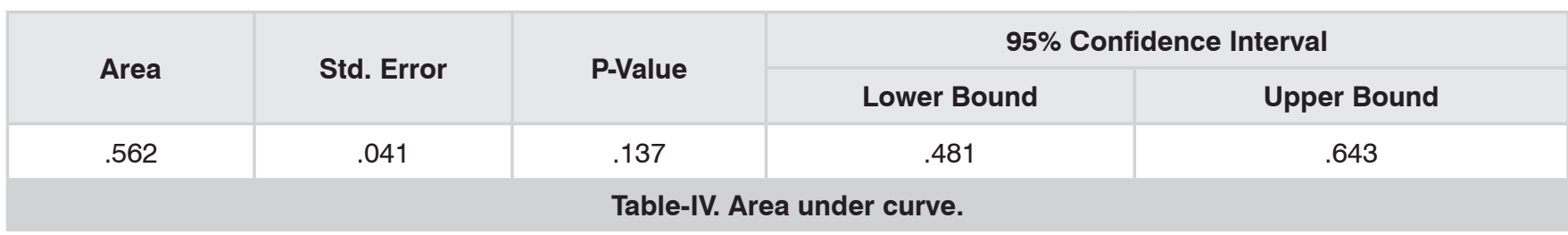




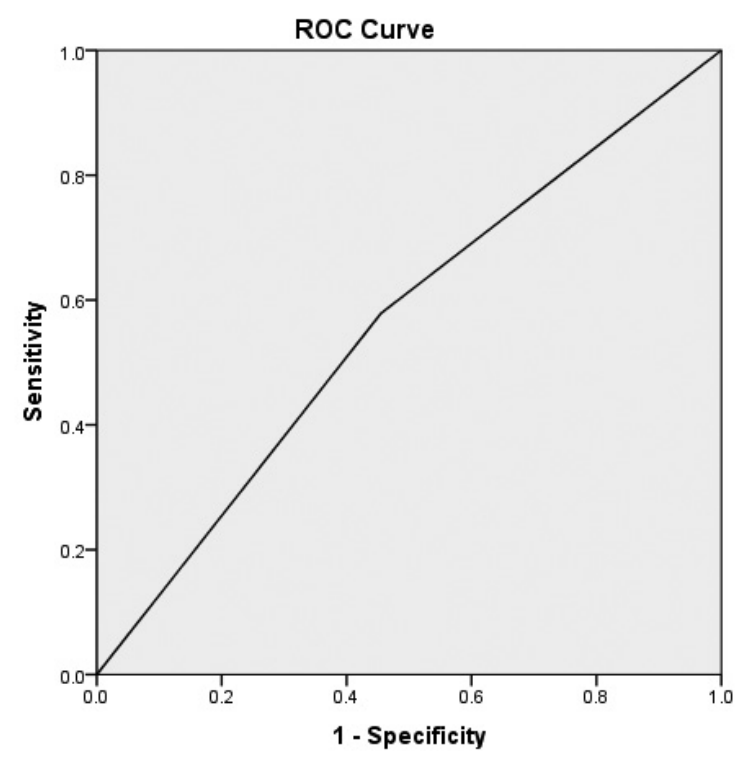

\section{DISCUSSION}

Presence of Bacteria in blood stream is called Bacteremia. Bacteremia and sepsis are accompanied by serious systemic illness, while occult bacteremia is an asymptomatic condition based upon absence of clinical toxicity. A study published in early 1970s, showed that 30 out of 111 children suffering from pneumococcal bacteremia had no identifiable focus of infection. ${ }^{8}$

To find any reliable predictors of bacteremia or occult bacteremia and its management, multiple studies had been conducted on febrile children but results are controversial. Although viral infections which are self-limiting are major cause of illness in febrile children, but there is still risk of occult bacteremia in these children. Studies found that febrile children of 3-36 months of age who have no identifiable focus of infection were found to have occult bacteremia with ranges between $1.0 \%-10.4 \% .^{9}$

Often, in healthy children, bacteremia is a selflimiting condition, but if left untreated, $10 \%$ of them may develop, meningitis, osteomyelitis, septic arthritis and pneumonia. According to the guidelines, management of febrile children include certain lab tests, antibiotics and hospitalization. Beside this, costs of these blood tests, unnecessary oral/ Iv therapy, adverse effects of antibiotics, increased medical treatment duration, increased work load of physician and laboratory, and emotional distress, all produce discomfort to parents and children. ${ }^{8}$

Fever phobia' is the result of physician's attitude of treating all febrile children with antibiotics, which leads misuse of antibiotics. ${ }^{10}$ Physicians have to opt for one of the two approaches to treat a febrile child; (1) Risk minimizer approach (2) Test minimizer approach. However, neither approach is better than other. ${ }^{8,9}$

Hence several tests like White Blood Cell count, C-Reactive Proteins, Procalcitonin have been studied along with some acute illness observation scores. One of them is Yale Observation score. ${ }^{8}$

Parameters of YOS are based upon signs and symptoms of basic vital functions and organ dysfunctions of a febrile child, which can be due to any cause. Moreover, overall condition of a febrile child can also be influenced by a lot of external factors that ultimately affect YOS. Hence YOS has a high negative predictive value, that is, it is a good scale to rule out bacteremia. But a positive YOS should be cautiously interpreted and investigated further. So YOS is very good for triage in an emergency department. ${ }^{7}$

The results of my study showed among all children with mean age of $12.9 \pm 8.24$ months, majority of the patients $(53.3 \%)$ had age $<1$ year. $52.4 \%$ patients were male. Sensitivity of YOS $>10 \%$ was noted to be $90.63 \%$, specificity was $79.45 \%$ with PPV and NPV was $65.91 \%$ and $95.08 \%$ respectively. Diagnostic accuracy was $82.86 \%$.

Bang et $\mathrm{al}^{7}$, concluded from his study done on febrile children that YOS is a simple, cost effective, and easily applicable test to predict bacteremia. His study was done on children of age 3-36 months and had sensitivity $87.93 \%$, specificity 83.78\%, NPV 94.66\%, PPV 68\%.

Similarly, McCarthy et $\mathrm{al}^{11}$ found that YOS if used before taking history and examinations of serious febrile children is a reliable test with a sensitivity of $77 \%$, specificity of $88 \%$, PPV of $56 \%$ and NPV of $97.3 \%$, and its sensitivity increased when 
combined with history and examination.

YOS was applied to febrile infants of age 4-8 weeks to detect serious illness. It showed sensitivity of $33 \%$, specificity of $73 \%$, PPV of $11 \%$ and NPV of $90 \%$. Hence Baker MD et $\mathrm{al}^{12}$ concluded YOS alone is insufficient to detect serious bacteremia in infants of 1-2-month age.

A study on factors predicting occult bacteremia in young children by Jamuana et $\mathrm{al}^{13}$ showed sensitivity of YOS was $100 \%$, specificity of $41.6 \%$, PPV of $6.6 \%$ and NPV of $100 \%$.

\section{CONCLUSION}

YOS > 10 is to be an easy, simple and applicable in diagnosis of bacteremia in febrile children. It can be used in routine clinical practice in rural area also for the evaluation of bacteremia. So, by using this clinical score bacteremia can be diagnosed and managed early and morbidity and mortality will be decreased.

Copyright@ 12 June, 2019.

\section{REFERENCES}

1. Biondi E, Evans R, Mischler M, Bendel-Stenzel M, Horstmann S, Lee V, et al. Epidemiology of bacteremia in febrile infants in the United States. Pediatrics. 2013; 132(6):990-6.

2. Steele RW. Fever with bacteremia: A disappearing classic. Consultant for Pediatr. 2013; 12:19-23.

3. Irwin AD, Drew RJ, Marshall P, Nguyen K, Hoyle E, Macfarlane KA, et al. Etiology of childhood bacteremia and timely antibiotics administration in the emergency department. Pediatrics. 2015; 135(4):63542.

4. Greenhow TL, Hung YY, Herz AM. Changing epidemiology of bacteremia in infants aged 1 week to 3 months. Pediatrics. 2012; 129(3): e590-6.
5. Chase M, Klasco RS, Joyce NR, Donnino MW, Wolfe RE, Shapiro NI. Predictors of bacteremia in emergency department patients with suspected infection. Am J Emerg Med. 2012; 30(9):1691-97.

6. Nigrovic LE, Mahajan PV, T zimenatos L, Alpern ER, Rogers AJ, Simmons T, et al. The accuracy of the yale observation scale score and unstructured clinician suspicion to identify febrile infants aged $\leq 60$ days with serious bacterial infections. Ann Emerg Med. 2015;66(4):86

7. Bang A, Chaturvedi P. Yale observation scale for prediction of bacteremia in febrile children. Indian J Pediatr. 2009; 76(6):599-604.

8. Yilmaz HL, Yildizdas RD, Alparslan N, Ozcan K, Yaman A, Kibar F. Screening tools for bacteraemia in a selected population of febrile children. Ann Acad Med Singapore. 2008; 37:192-9.

9. Isaacman DJ, Shults J, Gross TK, Davis PH, Harper M. Predictors of bacteremia in febrile children 3 to 36 months of age. Pediatrics. 2000; 106:977-82.

10. Akpede GO1, Akenzua Gl. Aetiology and management of children with acute fever of unknown origin. Paediatr Drugs. 2001; 3:169-93.

11. McCarthy PL, Sharpe MR, Spiesel SZ, Dolan TF, Forsyth BW, DeWitt TG, et al. Observation scales to identify serious illness in febrile children. Pediatrics. 1982; 70:802-9.

12. Baker MD, Avner JR, Bell LM. Failure of infant observation scales in detecting serious illness in febrile, 4- to 8-week-old infants. Pediatrics. 1990; 85:1040-3.

13. Jamuna R, Srinivasan $S$, Harish BN. Factors predicting occult bacteremia in young children. Indian $J$ Pediatr. 2000; 67:709-11. 


\section{AUTHORSHIP AND CONTRIBUTION DECLARATION}

\begin{tabular}{|c|l|l|l|}
\hline Sr. \# & \multicolumn{1}{|c|}{ Author(s) Full Name } & \multicolumn{1}{|c|}{ Contribution to the paper } & Author(s) Signature \\
\hline 1 & Motia Javed & 1st Author & \\
2 & Mehboob Alam Siddiqui & 2nd Author \\
3 & Zahid Mahmood Anjum & 3rd Author & 4th Author \\
4 & Fazal Elahi Bajwa & 5th Author & Hina Ayesha
\end{tabular}

